\title{
Initial Assessment of Radiometric Performance of N20 VIIRS Reflective Solar Bands Using Vicarious Approaches
}

\author{
A. $\mathrm{Wu}^{\mathrm{a}}$, T. Chang ${ }^{\mathrm{a}}, \mathrm{X} . \mathrm{Xiong}^{\mathrm{b}}$, and C. $\mathrm{Cao}^{\mathrm{c}}$ \\ ${ }^{a}$ The Science and Systems Applications, Inc., Lanham, MD 20706 \\ ${ }^{\mathrm{b}}$ Science and Exploration Directorate, NASA/GSFC, Greenbelt, MD 20771 \\ 'NOAA/NESDIS/STAR, College Park, MD 20740
}

\begin{abstract}
The newly launched (November 18, 2017) polar-orbiting satellite of the Joint Polar Satellite System (JPSS1), now transitioned to NOAA-20, is the follow-on mission to the SNPP (Suomi National Polar-orbiting Partnership) satellite, launched six years ago. NOAA-20 leads SNPP by a half orbit or about 50 minutes. The Visible Infrared Imaging Radiometer Suite (VIIRS) is a key sensor onboard both NOAA-20 and SNPP spacecraft with nearly identical band spectral responses. Similar to the heritage sensor MODIS, VIIRS has on-board calibration components including a solar diffuser (SD) and a solar diffuser stability monitor (SDSM) for the reflective solar bands (RSB), a V-groove blackbody for the thermal emissive bands (TEB), and a space view (SV) as background reference for calibration. This study provides an initial assessment of calibration of the NOAA-20 VIIRS reflective solar bands (RSB) by inter-comparison with measurements from SNPP VIIRS using various vicarious approaches. The first approach is based on a double difference method using observations from simultaneous nadir overpasses (SNO) with Aqua MODIS. The second is from the collected reflectances over the widely used Liby-4 desert site from 16-day repeatable orbits so each data point has the same viewing geometry relative to the site. The third approach is to use the frequent overpasses over the Dome C snow site. Results of this study provide useful information on NOAA-20 VIIRS post-launch calibration assessment and preliminary analysis of its calibration stability and consistency for the first 6 months.
\end{abstract}

Keywords: VIIRS, calibration, reflective solar bands, reflectance, inter-comparison

\section{INTRODUCTION}

The newly launched (November 18, 2017) JPSS-1 polar-orbiting satellite, now named to NOAA-20, is the follow-on mission to the SNPP satellite, launched six years ago on October 28, $2011^{1}$. Both satellites are in the afternoon orbit ( 1:30 PM local equator crossing time at ascending mode) and NOAA-20 leads SNPP by a half orbit or about 50 minutes. VIIRS is the primary sensor of the five sensors onboard both the NOAA20 and SNPP spacecraft ${ }^{2-4}$. There are 22 spectral bands with wavelengths ranging from 0.41 to $12.0 \mu \mathrm{m}$ including 14 reflective solar bands (RSB), 7 thermal emissive bands (TEB), and 1 day-night band (DNB). Each scan covers a $3000 \mathrm{~km}$ swath with spatial resolutions of $375 \mathrm{~m}$ and $750 \mathrm{~m}$ at nadir for the imaging 
bands (I-bands) and moderate resolution bands (M-bands), respectively, to provide imagery and radiometric measurements of the land, atmosphere, cryosphere, and oceans ${ }^{5-9}$.

VIIRS was developed with a close design heritage to MODIS of the NASA's EOS (Earth Observing System), currently operating on both Terra and Aqua spacecraft ${ }^{10-11}$. Both VIIRS and MODIS onboard calibrators include a solar diffuser (SD), a solar diffuser stability monitor (SDSM) and a blackbody (BB). A space view (SV) port provides measurements of sensor's background and signal offsets. VIIRS uses a rotating telescope assembly (RTA) compared to the scan mirror used in MODIS. Major advantages of RTA are better control of stray light, smaller range in angle of incidence to the half angle mirror (HAM), and better protection from contamination and degradation over time because all the optical elements are placed deep inside the instrument housing.

The production of NOAA-20 VIIRS SDR for the VIS and NIR bands started immediately after its nadir door's opening on December 13, 2017. The first TEB imagery was generated on January 8, 2018 after the SW/MWIR and LWIR focal planes were cooled down to the operation temperatures at $80.5 \mathrm{~K}$. Similar to SNPP VIIRS, during the first few months, NOAA20 VIIRS went through a series of Intensive Calibration and Validation (ICV) operational activities ${ }^{12}$. One of the ICV activities designed to support and improve on-orbit calibration of the RSB is a series of yaw maneuvers performed on January 25 and 26, 2018. The SDSM screen transmission derived from the yaw maneuver data provided noticeable improvement in the calculation of SD degradation ${ }^{13}$ and were used to replace the screen transmission based on prelaunch measurements. Starting from February 1, 2018, the NOAA-20 VIIRS SDR reached beta status. As a result of further changes and updates of look-up-tables (LUT), it was determined that the NOAA-20 VIIRS SDR achieved provisional status on March 20, 2018.

This study provides an initial assessment of the NOAA-20 VIIRS RSB calibration by inter-comparison with measurements from SNPP VIIRS using various vicarious approaches ${ }^{14-15}$. A close overlap of the two VIIRS spectral bands provides a unique opportunity to examine the newly launched sensor in reference the earlier one (Figure 1). The first approach is based on a double difference method using observations from simultaneous nadir overpasses (SNO) with Aqua MODIS. The second is from the collected reflectances over the widely used Libya-4 desert site from 16-day repeatable orbits so each data point has the same viewing geometry relative to the site. The third approach is to use the frequent overpasses over the Dome $\mathrm{C}$ snow site. The SNO approach allows a direct comparison between VIIRS and MODIS with the latter having been well calibrated and its performance well understood ${ }^{16}$. The two pseudo-invariant sites also provide a comparison between NOAA-20 and SNPP after a site-specific BRDF (bi-directional reflectance function) is applied to the observed reflectances by each sensor. Results of this study provide useful information on NOAA-20 VIIRS post-launch calibration stability and consistency during the early mission. The NOAA-20 and SNPP VIIRS SDR used in this study were generated by the NOAA Interface and Data Processing Segment (IDPS).

Section 2 provides a brief description of each of the three approaches for tracking the relative differences between NOAA-20 and SNPP VIIRS RSB and their stability monitoring. Section 3 provides the corresponding results from each approach, followed by discussion and summary in section 4 . 

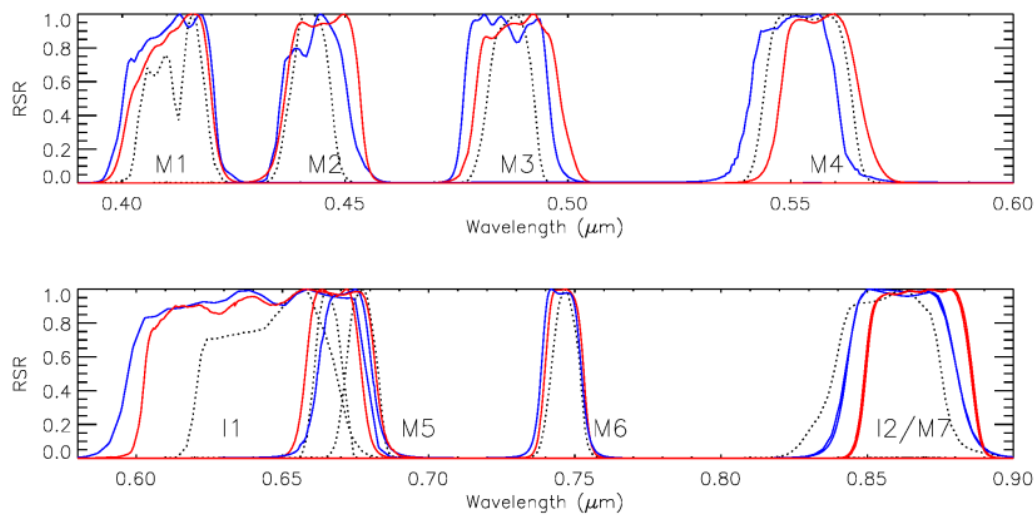

Figure 1. Comparison of relative spectral responses (RSR) among NOAA-20 VIIRS (solid red), SNPP VIIRS (solid blue) and Aqua MODIS (dotted black) for their spectrally matched VIS and NIR bands.

\section{METHODOLOGY}

\subsection{SNO}

The SNO method is based on a direct pixel level comparison between two crossover sensors. Since there is a close spectral overlap between VIIRS and Aqua MODIS (Figure 1) and their orbits are almost parallel to each other, this approach provides a large quantity of high quality inter-comparison data for the two sensors. There is an abundance of VIIRS and MODIS SNOs, with nearly one SNO event every three days. The closest time difference (less than 30 seconds) between VIIRS and MODIS occurs in the high latitude polar region. The SNO data sets collected in this study are all from the crossovers with less than 30 seconds. Figure 2 shows an example of a pixel-by-pixel comparison between VIIRS M1 and MODIS band 8 (0.412 um) reflectacnes obtained from the SNO event on August 2, 2018. A mean VIIRS to MODIS reflectance ratio, as shown in the figure, is calculated by the ensemble average of all collected VIIRS to MODIS reflectance pixel pairs. It should be noted that the comparison results from SNO data have uncertainties due to differences in VIIRS and MODIS pixel footprints. One method to reduce the impact due to the footprint difference is to increase the size of matching area from the pixel level to a large grid such as $1^{\circ}$ by $1^{\circ}$, which will significantly reduce the relative footprint difference. However, this method can only apply to a few of MODIS VIS and NIR bands because most ocean color bands are saturated over land and clouds. In order to limit the impact due to footprint difference on the mean of the VIIRS to MODIS reflectance ratio, a criterion of $2.5 \%$ for the relative standard deviation is used to select qualified SNO events. 


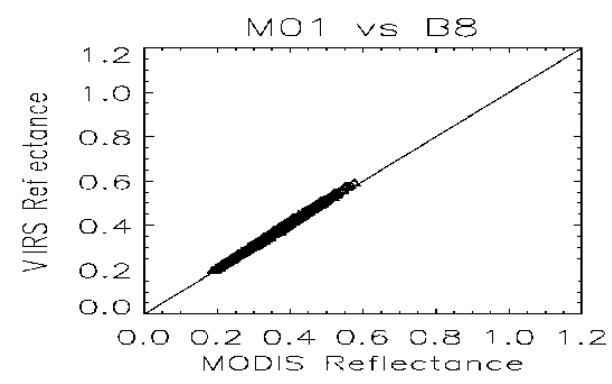

Figure 2. Comparison of NOAA-20 VIIRS M1 and Aqua MODIS band 8 SNO pixel reflectances based on data collected on August 2, 2018.

\subsection{Desert}

The Libya- 4 desert site $\left(28.5^{\circ} \mathrm{N}, 23.4^{\circ} \mathrm{E}\right)$ is one of best pseudo invariant desert sites selected to track sensor stability. The main reason that these desert sites are used is because of their high and nearly constant reflectance with little, if any, vegetation. Similar with the SNO approach, we choose near nadir overpasses with view zenith angles less than $5.0^{\circ}$ over the site, obtained from 16-day repeatable orbits. Examination of the reflectance trends show that there are still a small but noticeable seasonal pattern indicating that there is a need to correct for the BRDF impact. Since overpasses with a fixed near nadir view angle are selected, the only angular dependent parameter that affects the observed reflectances for the site is the solar zenith angle, assuming the impact due to azimuth angle is negligible. This allows us to determine a site dependent BRDF model with coefficients derived from a cumulative dataset typically for a one-year period to cover the seasonal cycle for the angular parameters. The standard error of the BRDF model is around $1 \%$ and the error increases with wavelength from the VIS to NIR region. Since the viewing and solar zenith angles of the VIIRS and MODS observations over the site can be selected to have a similar angular range, the derived BRDF model based on measurements from one sensor can be applied to measurements from another sensor. Our testing results showed that replacing the VIIRS-based BRDF model with the MODIS-based model achieved a similar angular correction. In order to compare the results from the Libya-4 site with those obtained from the SNO approach, the MODIS-based BRDF model is used to correct VIIRS reflectances.

\subsection{Dome C}

The Dome $\mathrm{C}$ site $\left(75.3^{\circ} \mathrm{S}, 123.4^{\circ} \mathrm{E}\right)$ is also a pseudo invariant site but covered by snow all year round. The atmosphere over the surface is extremely cold and dry, making it an excellent site to monitor sensor performance [26]-[27]. The disadvantage of the site is that there are only less than six months of daytime data each year. Since even in the Antarctic summer, solar zenith angles over Dome $\mathrm{C}$ are mostly larger than $50^{\circ}$, the impact of the surface BRDF on sensor measured reflectances is significantly larger than the desert sites located in the Northern Africa Region. However, the Dome C reflectance factors follow a simple linear relationship with solar zenith angle which is shown in Figure 3a [28]. This provides a relatively simple 
approach to correct for the BRDF effect. Examination of the fitting residuals indicates that the linear relationship works better in the visible spectral region than the near-infrared region. Similar to the treatment of the results from the Libya-4 desert site, the MODIS-based BRDF models with coefficients derived using Dome C data from the Antarctic summer 2012 - 2013 are applied to the VIIRS reflectance data for the same site. Figure $3 \mathrm{~b}$ shows the BRDF corrected (MODIS-based BRDF) reflectance ratio as function of solar zenith angle for SNPP and NOAA-20 VIIRS M1, indicating the simple BRDF correction works well over solar zenith angles larger than $50.0^{\circ}$.

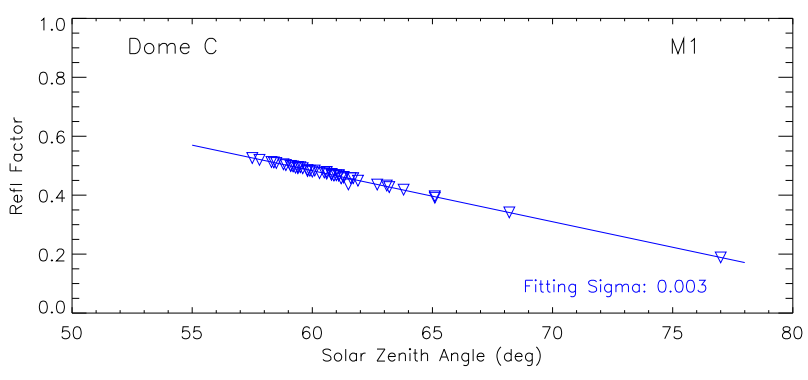

(a)

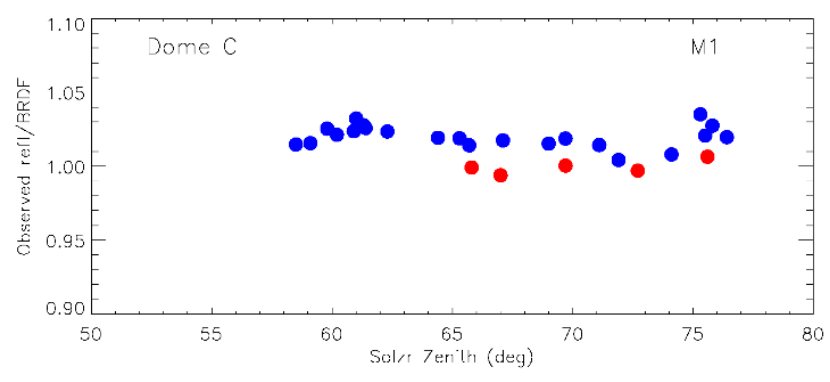

(b)

Figure 3. SNPP VIIRS reflectance factor versus solar zenith angle for M1 over the Dome $\mathrm{C}$ site. A line is determined from a linear fit to the data (a). Also shown are SNPP (blue) and NOAA-20 (red) reflectance factors normalized with the MODIS-based BRDF (b).

\section{RESULTS}

The averaged VIIRS to MODIS reflectance ratios from collected SNO events with a frequency of once every $5 \sim 6$ days are plotted versus time for the band M1 $(0.412 \mu \mathrm{m}), \mathrm{M} 4(0.555 \mu \mathrm{m}), \mathrm{I} 1(0.645 \mu \mathrm{m})$ and I2 $(0.865$ $\mu \mathrm{m})$ in Figure 4. Results from early mission for SNPP VIIRS are included to compare with those from the newly launched NOAA-20 VIIRS. The M1 data from SNPP with a longer period shows an apparent seasonal fluctuation, which is likely due to some residual BRDF effect. For NOAA-20 VIIRS, trends of M1-M3 are inconsistently higher during the first three months after the nadir door opened on December 13, 2017. This is due to inaccurate estimate of the initial SD degradation and similar results were also observed for SNPP VIIRS during the initial post-launch period. A much improved estimate of the degradation, particularly for the shortest wavelength bands, was obtained with the NOAA-20 yaw maneuver and more cumulative onorbit SD data. The noticeable drop in reflectance for M1 (close to the end of March 2018) as shown in Figure 4 is due to a major update of the SDSM screen transmittance table used to determine the SD degradation. It is noticed that NOAA-20 reflectances are lower than SNPP, on the order of 1 to $4 \%$. The reason for the systematic differences is still unknown, since a rapid post-launch degradation in the SD BRDF mostly occurs in the blue wavelength region. 

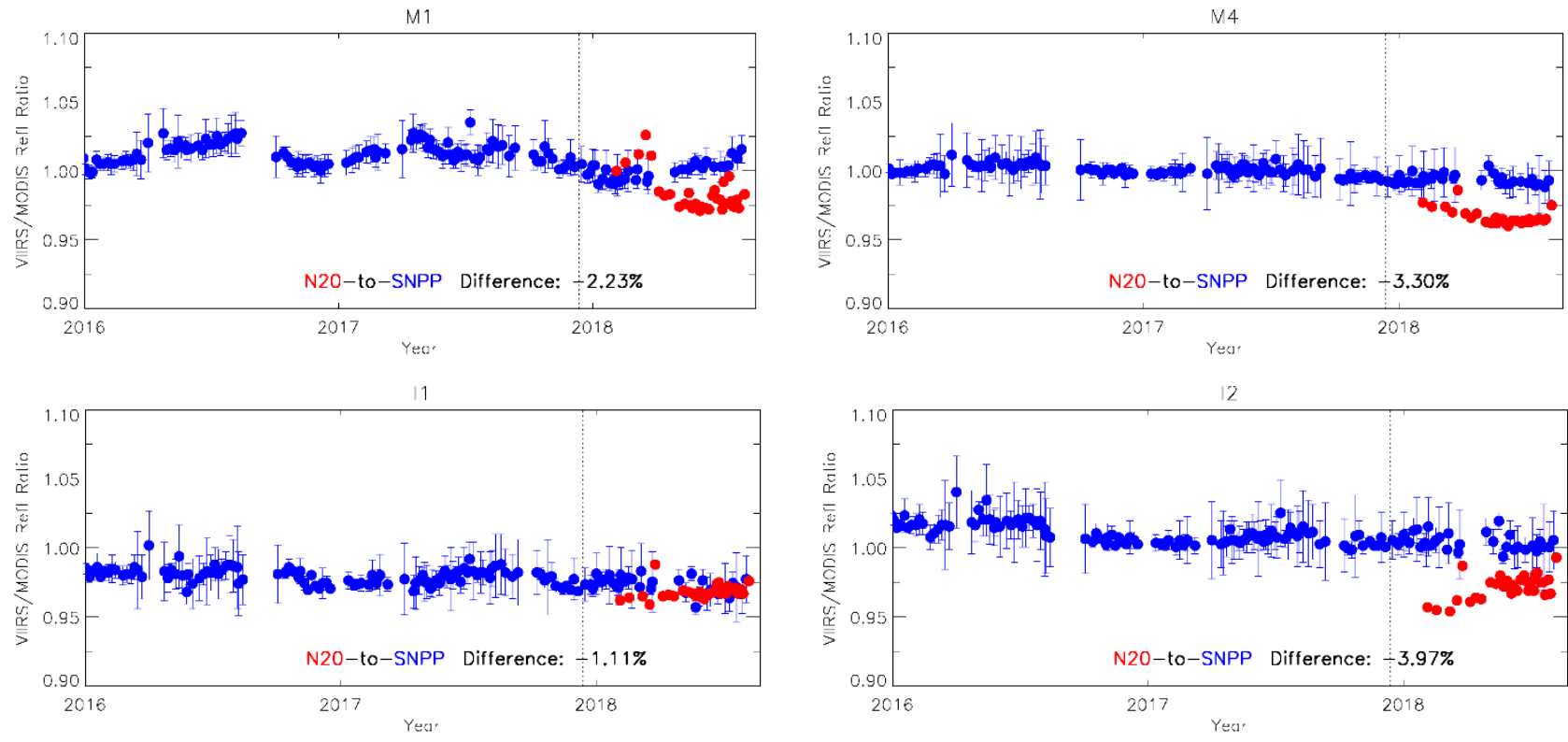

Figure 4. Trends of VIIRS to MODIS reflectance ratios for M1, M4, I1 and I2 obtained from SNOs. Results from NOAA-20 (red) and SNPP (blue) VIIRS are referenced to Aqua MODIS.

For the Libya-4 desert site, the VIIRS reflectance trends are derived after normalization with the MODISbased BRDF, as shown in Figure 5. For SNPP, results indicate that VIIRS reflectances are stable to within $1.0 \%$. Comparison of the stability results obtained between the SNO approach and the Libya-4 desert site shows that the latter method is more suitable to track sensor stability, as expected. Similarly, trends of M1M3 for NOAA-20 are inconsistently higher during the initial post-launch period mainly due to the use of the prelaunch-based SDSM screen transmittance table. Comparison of the relative differences between SNPP and NOAA-20 from SNO and the desert site shows that they are in agreement to within $2 \%$, except for M4, where the two approaches are inconsistent.
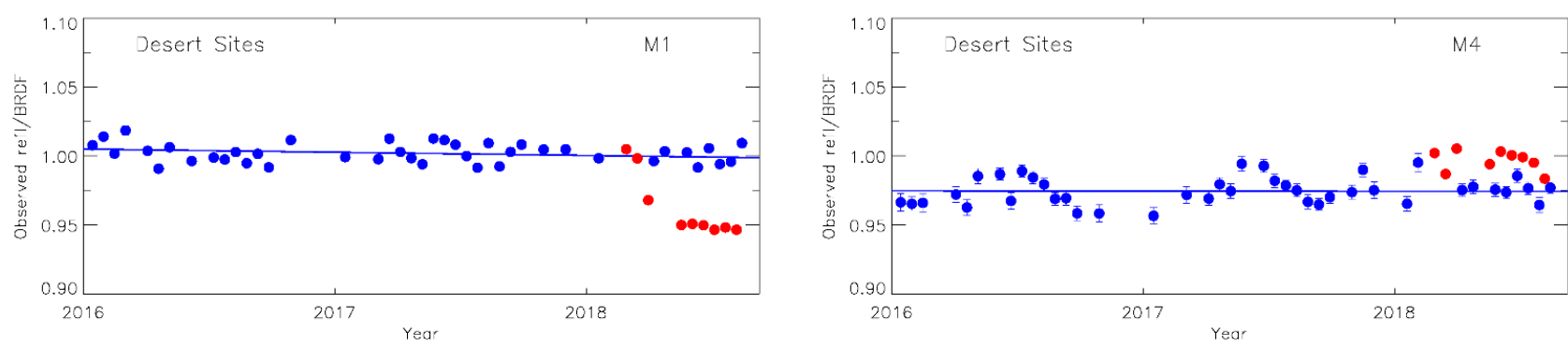

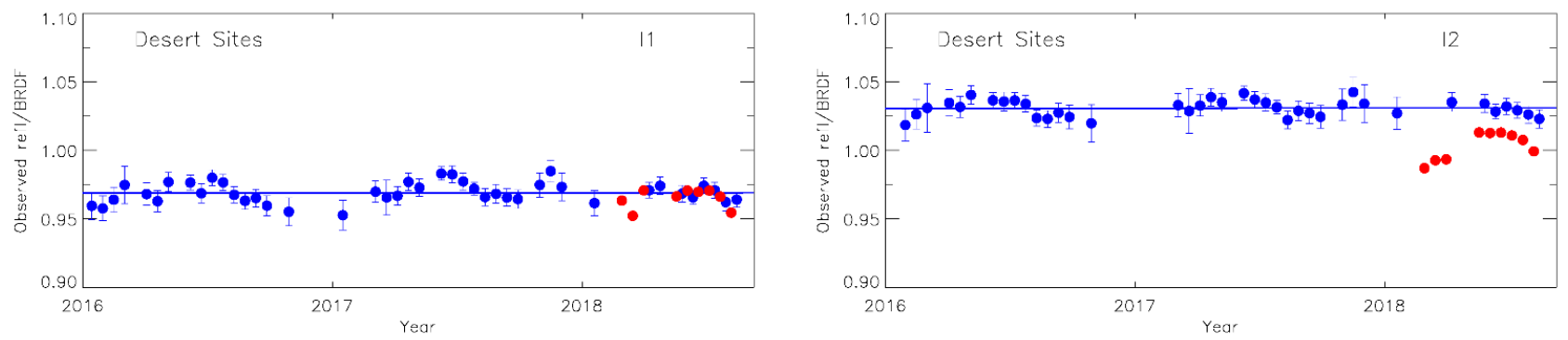

Figure 5. Trends of NOAA-20 (red) and SNPP (blue) VIIRS reflectance normalized with the MODIS-based BRDF for M1, M4, I1 and I2 obtained from 16-day repeatable nadir overpasses over the Libya-4 desert site. Lines are determined from the linear-fit to SNPP VIIRS data.

The trends for the Dome $\mathrm{C}$ site are shown in Figure 6. By comparison with the trends from the Libya-4 desert site, they are associated with relatively large variations due to errors in the BRDF fitting. The mean reflectance differences between the two VIIRS sensors are generally consistent with those from the SNO and desert approaches. Since available overpasses over the Dome C site were from the Antarctic summer, there were a few data points early in the mission for NOAA-20 VIIRS. As discussed earlier in this section, results for M1-M3 during this period were not stable because of errors in the initial calibration.
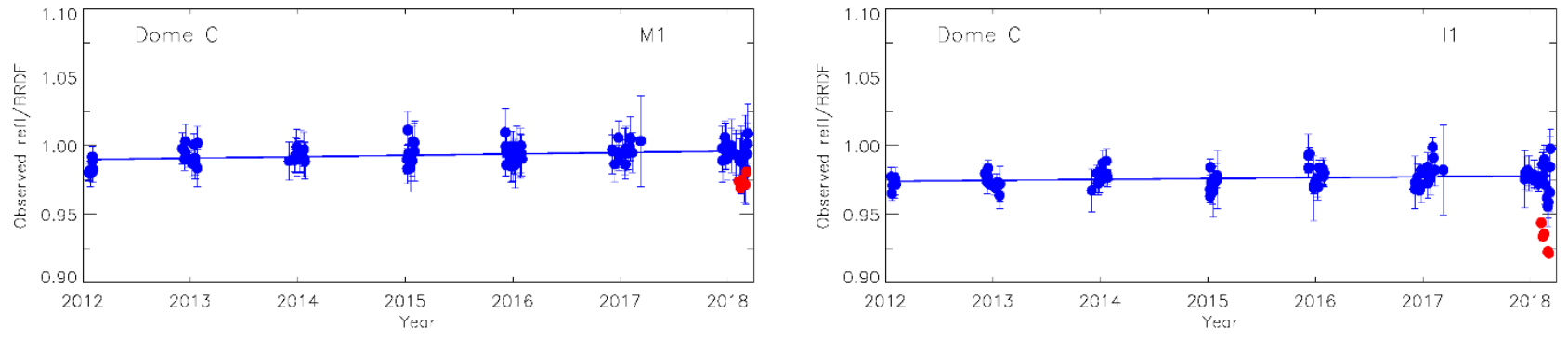

Figure 6. Trends of NOAA-20 (red) and SNPP (blue) VIIRS reflectance normalized with the MODIS-based BRDF for M1 and I1 obtained from near nadir overpasses over the Dome C snow site. Lines are determined from the linear-fit to SNPP VIIRS data.

Table 1 lists the mean differences between N20 and SNPP VIIRS for M1-M5, M7, I1-I2. Result for M6 is not included due to a saturation issue for the corresponding MODIS band. It is interesting to note that except for M4 for the Libya-4 site, all results are negative mostly ranging from -2 to $-4 \%$, indicating NOAA-20 VIIRS is consistently lower than SNPP for the visible and near infrared region. Errors in the calculation of the SD on-orbit degradation are unlikely the reason for the systematic bias, since the degradation occurs mainly in the blue region. Further analysis of prelaunch data is worth considering the fact that the VIIRS RSB is referenced to the at-launch baseline calibration results, which are obtain from prelaunch characterization. The exceptional positive bias for M4 over the Libya-4 desert site is further studied by examining the difference caused by RSR using MODTRAN simulations ${ }^{17}$ and SCIAMACKY (Scanning Imaging Absorption Spectrometer for Atmospheric Chartography) observations ${ }^{18}$, one of ten instruments 
aboard of ESA's Environmental Satellite, ENVISAT. This is because there are two considerations. First, the central wavelength shift for M4 between SNPP and NOAA-20 is the largest, as shown in Figure 1. Second, the integrated total out-of-band (OOB) response in M4 for SNPP is also the largest among all RSB. Table 2 compares theoretical VIIRS-to-MODIS radiance ratios between SNPP and NOAA-20 for four different underlying surfaces (ocean, desert, snow and cloud). Results from MODTRAN simulations show that the variations in ratio are less than $1 \%$ for the four surfaces, while results from the SCIAMACKY data are over 3\%, as highlighted in the table. A slightly large variation in SNPP is expected since there is a significant OOB contribution in M4. Taking a ratio of NOAA-20 divided SNPP by using results from the SCIAMACKY data, it is shown that the ratio for the desert site (last column if Table 2) is at least 3.5\% higher than the other three surfaces (ocean, snow and cloud). This is consistent with the observed positive bias for M4 over the Libya-4 desert site in comparison with the results from SNO and Dome C.

Table 1. Averaged NOAA-20 and SNPP VIIRS reflectance differences from the three vicarious approaches (SNO, Desert and Dome C) for selected VIS and NIR bands. The difference is derived in percentage $(\%)$ by subtracting the NOAA-20 reflectances from the SNPP values.

\begin{tabular}{|c|c|c|c|}
\hline Band & $\begin{array}{c}\text { SNO } \\
(\%)\end{array}$ & $\begin{array}{c}\text { Desert } \\
(\%)\end{array}$ & $\begin{array}{c}\text { Dome C } \\
(\%)\end{array}$ \\
\hline M1 & -2.2 & -4.3 & -2.0 \\
\hline M2 & -1.8 & -1.7 & n/a \\
\hline M3 & -1.7 & -1.4 & -3.6 \\
\hline M4 & -3.3 & 2.2 & -5.6 \\
\hline M5 & -2.6 & -3.2 & -4.3 \\
\hline M7 & -3.9 & -2.4 & -5.4 \\
\hline I1 & -1.1 & -0.5 & -4.6 \\
\hline I2 & -4.0 & -2.7 & -6.5 \\
\hline
\end{tabular}

Table 2. SNPP and NOAA-20 VIIRS to MODIS reflectance ratios for M4, determined using MODTRAN (v.5.2.0.0) simulations and SCIAMACKY hyperspectral observations for four surfaces (Ocean, Desert, Snow and Cloud). The SNPP VIIRS SDR solar irradiance model based on MODTRAN v4.3 was used.

\begin{tabular}{|l|l|l|l|l|l|l|}
\hline \multirow{2}{*}{$\begin{array}{l}\text { Surface } \\
\text { Type }\end{array}$} & \multicolumn{2}{|c|}{ MODTRAN } & \multicolumn{3}{c|}{ SCIAMACKY } \\
\cline { 2 - 7 } & SNPP & N20 & N20/SNPP & SNPP & N20 & N20/SNPP \\
\hline
\end{tabular}




\begin{tabular}{|l|l|l|l|l|l|l|}
\hline Ocean & 1.008 & 0.992 & 0.984 & 1.031 & 0.983 & 0.953 \\
\hline Desert & 1.000 & 0.999 & 0.999 & 0.996 & 1.013 & 1.017 \\
\hline Snow & 1.006 & 0.994 & 0.988 & 1.013 & 0.995 & 0.982 \\
\hline Cloud & 1.005 & 0.995 & 0.990 & 1.020 & 0.989 & 0.970 \\
\hline
\end{tabular}

\section{SUMMARY}

This study provides an initial assessment of the NOAA-20 VIIRS RSB calibration by inter-comparison with measurements from SNPP VIIRS using various vicarious approaches (SNO, desert and Dome C). Results from the Dome $\mathrm{C}$ approach are less reliable than the other two approaches due to limited overpasses in the Antarctic summer and errors in the initial calibration for a few shortest wavelength bands. Results show that except for M4 for the desert site due to a large RSR impact, NOAA-20 is consistently lower than SNPP from -2 to $-4 \%$ for the visible and near infrared spectral region. The exceptional positive bias for M4 over the desert site is due to the existing RSR differences between SNPP and NOAA-20 based on the simulated radiance differences using SCIAMACKY hyperspectral observational data. These results suggest a further investigation of prelaunch test data is worth considering the fact that the VIIRS RSB at-launch baseline calibration is referenced to the prelaunch characterization. More on-orbit data is required to have reliable analysis of NOAA-20 calibration stability.

\section{ACKNOWLEDGMENT}

Jeff McIntire from NASA VIIRS Characterization Support Team (VCST) provided useful comments and grammatical corrections.

\section{REFERENCES}

1. Goldberg, M. D., H. Kilcoyne, H. Cikanek, and A. Mehta, Joint Polar Satellite System: The United States next generation civilian polar-orbiting environmental satellite system, Journal of Geophysical Research: atmospheres, Vol. 118, pp. 13,463-13,475, (2013).

2. Schueler, C. F., E. Clement, P. Ardanuy, C. Welsh, F. De Luccia, and H. Swenson, NPOESS VIIRS sensor design overview," Proc. SPIE, 4483, 11-23, (2002).

3. Murphy, R.P., P. E. Ardanuy, F. De Luccia, J. E. Clement, and C. Schueler, "The visible infrared imaging radiometer suite," in Earth Science Satellite Remote Sensing, vol. 1, New York, USA: Springer-Verlag, pp. 199-223, (2006).

4. Cao, C., F. De Luccia, X. Xiong, R. Wolfe, and F. Weng, "Early On-orbit Performance of the Visible Infrared Imaging Radiometer Suite (VIIRS) onboard the Suomi National Polar-orbiting 
Partnership (S-NPP) Satellite,” IEEE Trans. Geosci. Remote Sens., vol.52, no.2, pp.1142-1156, (2014).

5. Schueler, C. F., E. Clement, P. Ardanuy, C. Welsh, F. De Luccia, and H. Swenson, "NPOESS VIIRS sensor design overview," Proc. SPIE, vol. 4483, 11-23, (2002).

6. Murphy, R.P., P. E. Ardanuy, F. De Luccia, J. E. Clement, and C. Schueler, "The visible infrared imaging radiometer suite, Earth Science Satellite Remote Sensing," vol. 1, New York, USA: Springer-Verlag, pp. 199-223, (2006).

7. [6] Justice, C. O., M. O. Rom’an, I. Csiszar, E. F. Vermote, 570 R. E. Wolfe, S. J. Hook, M. Friedl, Z. Wang, C. B. Schaaf, T. Miura, M. Tschudi, G. Riggs, D. K. Hall, A. I., Lyapustin, S. Devadiga, C. Davidson, and E. J. Masuoka, "Land and cryosphere products from Suomi NPP VIIRS: Overview and status," Journal of Geophysical Research (Atmospheres), 118, 9753-9765, doi:10.1002/jgrd.50771, (2013).

8. Wang, M., X. Liu, L. Tan, L. Jiang, S. Son, W. Shi, K. Rausch, and K. Voss, "Impacts of VIIRS SDR performance on ocean color products," Journal of Geophysical Research (Atmospheres), 118, 10,347, doi:10.1002/jgrd.50793, (2013).

9. Liu, H., L. A. Remer, J. Huang, H.-C. Huang, S. Kondragunta, I. Laszlo, M. Oo, and J. M. Jackson, Preliminary evaluation of S-NPP VIIRS aerosol optical thickness, Journal of Geophysical Research (Atmospheres), 119, 3942-3962, (2014).

10. Salomonson, V., W. Barnes, X. Xiong, S. Kempler, and E. Masuoka, "An overview of the Earth Observing System MODIS instrument and associated data systems performance," Proc. IEEE Int. Geosci. Remote Sens. Symp., pp. 1174-1176, (2002).

11. X. Xiong, K. Chiang, J. Esposito, B. Guenther, and W. L. Barnes, "MODIS On-orbit Calibration and Characterization," Metrologia 40 89-92, (2003).

12. Butler, J., X. Xiong, A. B. Barnes, F. S Patt, J. Sun, and K. Chiang, “An overview of Suomi NPP VIIRS calibration maneuvers", Proc. SPIE 8510, Earth Observing Systems XVII, 85101J, (2012).

13. McIntire, J., D. Moyer, B. Efremova, H. Oudrari, and X. Xiong, "On-Orbit Characterization of SNPP VIIRS Transmission Functions," IEEE Transactions on Geoscience and Remote Sensing, vol. 53, issue 5, pp. 2354-2365, (2015).

14. Wu, A., X. Xiong, D. R. Doelling, D. Morstad, A. Angal, and R. Bhatt, "Characterization of Terra and Aqua MODIS VIS, NIR, and SWIR Spectral Bands' Calibration Stability", IEEE Transactions on Geoscience and Remote Sensing, vol. 51, issue 7, 4330-4338, (2013).

15. Wu, A., X. Xiong, C. Cao, and K. Chiang, "Assessment of SNPP VIIRS VIS/NIR Radiometric Calibration Stability Using Aqua MODIS and Invariant Surface Targets", IEEE Transactions on Geoscience and Remote Sensing, vol. 54, issue 5, pp. 2918-2924, (2016).

16. Cao, C., Weinreb, M. and Xu, H., "Predicting simultaneous nadir overpasses among polar-orbiting meteorological satellites for intersatellite calibration of radiometers," Journal of Atmospheric and Oceanic Technology, vol. 21, 537-542, (2004).

17. A. Berk, G. P. Anderson, P. K. Acharya, and E. P. Shettle, MODTRAN 5.2.0.0 User's Manual Air Force Res. Lab., Space Veh. Directorate, Air Force Materiel Command, Bedford, MA, USA, 01731-3010, (2008).

18. Gottwald, M., and H. Bovensmann, "SCIAMACHY - Exploring the Changing Earth's Atmosphere", Published by Springer, New York, (2011). 